\title{
Results from an Investigation into Extra-Vehicular Activity (EVA) Training related Shoulder Injuries
}

\author{
Brian J. Johnson* \\ NASA Lyndon B. Johnson Space Center, Houston, TX, 77058 \\ and \\ David Williams, $\mathrm{MD}^{\dagger}$ \\ Canadian Space Agency, Saint-Hubert, Quebec, Canada
}

\begin{abstract}
The number and complexity of extravehicular activities (EVAs) required for the completion and maintenance of the International Space Station (ISS) is unprecedented. The training required to successfully complete this magnitude of space walks presents a real risk of overuse musculoskeletal injuries to the EVA crew population. There was mounting evidence raised by crewmembers, trainers, and physicians at the Johnson Space Center (JSC) between 1999 and 2002 that suggested a link between training in the Neutral Buoyancy Lab (NBL) and the several reported cases of shoulder injuries. The short- and long-term health consequences of shoulder injury to astronauts in training as well as the potential mission impact associated with surgical intervention to assigned EVA crew point to this as a critical problem that must be mitigated. Thus, a multi-directorate tiger team was formed in December of 2002 led by the EVA Office and Astronaut Office at the JSC. The primary objectives of this Tiger Team were to evaluate the prevalence of these injuries and substantiate the relationship to training in the NBL with the crewperson operating in the EVA Mobility Unit (EMU). Between December 2002 and June of 2003 the team collected data, surveyed crewmembers, consulted with a variety of physicians, and performed tests. The results of this effort were combined with the vast knowledge and experience of the Tiger Team members to formulate several findings and over fifty recommendations. This paper summarizes those findings and recommendations as well as the process by which these were determined. The Tiger Team concluded that training in the NBL was directly linked to several major and minor shoulder injuries that had occurred. With the assistance of JSC flight surgeons, outside consultants, and the lead crewmember/physician on the team, the mechanisms of injury were determined. These mechanisms were then linked to specific aspects of the hardware design, operational techniques, and the training environment. During the 1999 to 2003 time frame many variables converged to make it impossible to determine with any accuracy which one or two root causes were primarily involved. Therefore a broad range of recommendations was established to prevent future injury to crewmembers training in the NBL in the near term. Many of these recommendations are lessons learned that are essentially timeless and therefore should be passed on to future EVA endeavors to ensure that hardware designs and operational techniques utilized in the future consider the demands of training on the human body here on earth.
\end{abstract}

\section{Nomenclature}

$A S C R=$ Astronaut Strengthening, Conditioning and Rehabilitation (Team)

$E M U=$ Extra-vehicular Mobility Unit

FMC = Flight Medical Clinic

$H U T=$ Hard Upper torso

$N B L=$ Neutral Buoyancy Laboratory (located at the Sonny Carter Training Center in Houston)

*EMU Program Manager, EVA Office, 2100 NASA Parkway / XA, Senior Member.

${ }^{\dagger}$ Astronaut, Crew Office, 6767 Route De l'Aeroport 


\section{Introduction}

Key to the future of human space exploration is the ability for humans to leave the confines of a spacecraft by donning a space suit. The capability to perform Extra-vehicular Activity (EVA) has allowed for humans to come into more intimate contact with their surroundings (such as exploring the surface of the moon) as well as aiding the assembly and maintenance of large space structures and satellites. A vast majority of the EVAs performed today are in support of the assembly and maintenance of the International Space Station (ISS). These EVAs are performed in most current configuration of the United State's space suit called the Extra-vehicular Mobility Unit or the EMU. Between 1999 and 2003 (5 years), 39 EVAs were performed in the EMU, which is considerable when compared to a total count of 42 performed in the previous 18 years (1981 through 1998) of the Space Shuttle Program. In addition to the increase in quantity of EVAs, the complexity of these EVAs has also risen tremendously.

Commensurate with the increased EVA requirements (quantity and complexity) is the increased need for training and preparation. The training required to perform an EVA includes a significant amount of time obtain experience in the EMU. Suit experience is gained by donning training versions of the suit (usually downgraded flight EMUs) and performing simulation runs in the NBL. The NBL is essentially an extremely large swimming pool in which up to 5 suited crewmembers may experience a real time simulation of the space environment. The suited crewmembers are outfitted with weights to make them neutrally buoyant. Although suit is neutrally buoyant, the crewmember inside is not completely weightless and still contends with the effects of gravity (particularly if inverted) and the drag of the water while translating. Life-size mockups with hi-fidelity EVA interface hardware (incluciing ISS elements and the Space Shuttle) are placed in the pool to support the simulation of EVA tasks. Astronauts utilize the NBL to gain familiarization with the suit, the specific task at hand, and overall "weightless" environment. Performing an EVA in the EMU on-orbit can be a demanding physical task as substantiated by many experienced EVA veterans. Performing a simulation in the NBL can be even more demanding given the physics of the situation with increased suit torque and gravitational issues to be contended with.

An EVA crewmember selected for a mission requiring an EVA typically performs between 10 and 15 six-hour practice exercises in the NBL. This means, for example, that for a Shuttle mission with 3 scheduled EVAs, each crewmember must perform at least $30 \mathrm{NBL}$ runs in a time span of about 18 months. Concurrent with the peak in EVA training activities in 1999, issues with astronaut injuries began to surface. It was not until 2002 that enough evidence accumulated to suggest a link between EVA training and occurrence of major injuries with crewmembers. Most serious of the injury concerns were those requiring surgical intervention. It was at this time that at least two experienced EVA crewmembers who had recently completed missions had elected to have surgery to repair shoulder issues. With the evidence suggesting a link between EVA training and shoulder injury and the potential severity of some of these injuries the representatives for the Astronaut Office and the EVA Office promptly decided to charter a tiger team to investigate this possible link. This team was dubbed the "Shoulder Injury Tiger Team" and investigated this issue between December 2002 and June 2003. The detailed results of the Tiger Team were published in a NASA Technical Memorandum in June of 2003 titled "EMU Shoulder Injury Tiger Team Report" 1.

The focus of this paper will be to summarize the pertinent findings and recommendations that should be carried forward into future EVA and human space flight endeavors. These have been broken down into three categories, those that focus on the technical aspects of the issue, those that are related to health and medical monitoring, and those that focus on the cultural and organizational aspects. This paper will focus on a subset of the findings and recommendations in all three categories that may be beneficial to a wider audience. This paper will not replay the engineering and medical analysis used to develop more specific recommendations intended for a much more limited audience. That information can be found in the in the technical manual cited above and in the reference. It is the hope of the authors that this assessment represents one of the more significant chapters in the book of lessons learned in assembling and maintaining the International Space Station. Integrating humans and machines for the purposes of space exploration can and potentially will have a permanent affect on the health of future explorers. It is our intent here to communicate the lessons learned to future leaders of human space flight programs. 


\section{Investigation Process and Key Findings}

To address this issue a Tiger Team was established whose charter was to investigate the shoulder injury claims, determine if there indeed was a link between training for EVA missions and, if a link was established, determine a course for corrective action. This team was co-chaired by the authors, who represent the EVA Office and Crew Office at the Johnson Space Center (JSC). The team was comprised of representatives from several organizations at JSC including the Mission Operations Directorate, the Flight Medicine Clinic, the Space and Life Sciences Division (including Anthropometric - Biomechanic Facility and the Astronaut Strength, Conditioning and Rehabilitation office) and the Engineering Directorate. (Refer to the acknowledgment section for a detailed listing) In addition, several experts in the field of orthopedics and sports medicine were consulted. The team broke up into four subgroups to address this issue in a parallel fashion. These subgroups were Injury Survey/Post-dive Symptoms; Anthropometrics, Biomechanics and Suit Design; Conditioning and Rehabilitation Medicine; and EVA Skills/Mission Training. However, before each team could adequately examine this issue, the problem needed to be bounded. This required the collection of hard data. Since there was no one single reliable and current source for data injury data for crewmembers a survey was built and personally administered by Dr. Williams. Data was collected from 42 volunteer astronauts of the 104-member astronaut corps during the study. Because of time and logistics issues it was not possible to question the entire current cadre so the survey reflects a random selection of both EVA-experienced and non-EVA-experienced astronauts. Of the 42 astronauts interviewed, roughly half were considered EVA experienced (20). The survey collected various categories of data including subject baseline data (age, height, weight, etc.), EVA training experience, injury experience, and physical training habits. In addition, the survey asked the subjects for their personal opinion on causal mechanisms for injuries (if applicable) occurring during their mission training.

The results of the survey were quite enlightening and provided a backbone to many of the subsequent recommendations. The key findings from the survey are as follows:

- The physical demands of EVA training place the experienced EVA astronaut at increased risk of shoulder injury as the number of crew reporting shoulder pain during EVA training increased from astronaut candidates ( $0 \%$ ) to contingency ( $11 \%)$, development runs (37\%), EVA skills (45\%) to mission assigned training $(56 \%)$.

- Three of the astronauts in the study group have had surgery for shoulder injuries, however only two of these are considered EVA training-related, as the third astronaut had sustained shoulder injuries in a fall. Neither of the two cases of EVA training-related shoulder injury had a previous history of shoulder injuries prior to EVA training.

- The onset of shoulder pain during or within 24 hours of an NBL run strongly suggests a causal relationship in which some activity during the run precipitated the shoulder pain. Repeated episodes of shoulder pain during training suggest the evolution of overuse syndromes that could ultimately lead to lesions requiring surgical repair.

- Data was obtained on suspected causal mechanisms in 17 cases of EVA training-related shoulder pain (Refer to Table 2-6). In 8 of these cases, 22 multiple causes were listed in 7 different categories resulting in a total of 31 suspected causes. These are rank ordered in Table 1 below.

Table 1 - Possible Causal Mechanisms

\begin{tabular}{lcc}
\hline & EVA Crew & $\%$ \\
\hline Inverted Position Suspected Cause: & 8 & $25.8 \%$ \\
Planar HUT Suspected Cause: & 7 & $22.6 \%$ \\
Repetitive Motion: & 6 & $19.4 \%$ \\
Heavy Tools Suspected Cause: & 5 & $16.1 \%$ \\
Frequent NBL Runs: & 2 & $6.5 \%$ \\
Specific Arm Position: & 2 & $6.5 \%$ \\
EMU Donning: & 1 & $3.2 \%$ \\
\hline
\end{tabular}


Although it cannot be irrefutably proven, the survey results presented a strong case in linking EVA training with the onset of various shoulder injuries among EVA crewmembers. The survey findings were crucial to the investigation but alone were not enough to adequately address this issue. Dr. Williams, with the help of outside consultants, then established a classification of shoulder injury and educated the rest of the team on the biomechanical mechanisms for injury. Armed with the survey data and better understanding how shoulder injuries occur, each sub-team could then begin examining the protocols, procedures, hardware, and practices that might be linked to this issue. It should be noted here that at no time was there a "smoking gun" for the cause of shoulder injuries. This issue involved several variables and therefore a wide search for all the potential causes was required. Likewise, there would be many recommendations for corrective action. After several months of investigation the team had developed a long list of findings. In order for the reader to better understand the summarized findings a short background on the people, hardware and training are provided. It would be prohibitively long to characterize the detailed background and findings from each sub-team, including all the terminology and names used within the findings. Therefore only a short synopsis below has been provided to give the reader a flavor of the multi-faceted nature of this issue. A full description of these details is provided in the technical memorandum published by the Tiger Team .

People / Organizations: There are several organizations responsible for supporting and conducting EVA training at the Johnson Space Center. The EVA Office provides the EVA hardware requirements and has overall authority on mission planning. The Mission Operations Directorate is responsible for developing and implementing the training required to perform an EVA. The ASCR team representatives provide a location (gym) and physicaltrainers to support overall crewmember conditioning. The Engineering Directorate provides support for flight andtraining hardware development. The Space and Life Sciences Directorate oversees Medical Operations, which provides the crew with dedicated Flight Surgeons, and the Anthropometric and Biomechanical Facility, which performs research and evaluation of flight equipment, procedures, and systems from the perspective of biomechanics, human performance, and ergonomics.

Hardware: The hardware component that received the most attention in this investigation was the Hard Upper Torso, or HUT for short, which is part of the EMU (see Figure 1). The HUT is essentially a fiberglass shell that provides pressure retention and structural attachment points for the helmet, arms and waist and the Primary Life Support System (PLSS). The flight and training versions (those used in the NBL) are identical. Primary design drivers for a HUT were that it had to be adequately sized to provide efficient mobility and allow for ease of suit entry (don) and exit (doff) for the crewman. Today three sizes of HUTs exist to support the crew cadre: medium, large and extra large. Two HUT configurations were certified for the Shuttle Program. These are referred to as the Pivoted HUT and the Planar HUT. The Pivoted HUT was the original design but was phased out between 1997 and 2000 when the Planar HUT was put into service.

The key difference between the two configurations is with the attachment design between the arm and the HUT. In the Pivoted HUT, the scye, or shoulder, bearing attached to gimbals allowing for rotation about 2 axes. The Planar HUT scye bearing is fixed and "pivot-less". The Pivoted HUT performed extremely well since the gimbaled design allowed for an easier don/doff capability and enhanced overall mobility during operation. The gimbal design in the Pivoted HUT, however, was found to not be robust and represented a catastrophic hazard if it failed. Ultimately, a failure of a HUT pivot during a manned test led to the quick development of a replacement design, dubbed the Planar HUT. Without the gimbal pivot feature the scyes, or arm openings, in the Planar HUT were optimized to afford the greatest possible mobility without overly impairing the donning envelope. This compromise resulted in a decrease in overhead and downward reach in the Planar HUT. In addition, a Program decision was made during the design phase to accommodate all crewmembers in two sizes (medium and large) of Planar HUTs. This decision required minor, but important, dimensional changes to the HUT so that the Planar and Pivoted HUT sizes were no longer analogous. Later, a third size Planar HUT was added to the inventory to address fit issues associated with the crewmembers who fit in the Extra-Large Pivoted HUT. All of this historical information is pertinent since many of the current crew cadre were originally sized into Pivoted HUTs and trained in this design through the late 1990s prior to shifting over to the newer Planar design. 
Figure 1 - Planar HUT (left) and EMU without garment on the Planar HUT (right)
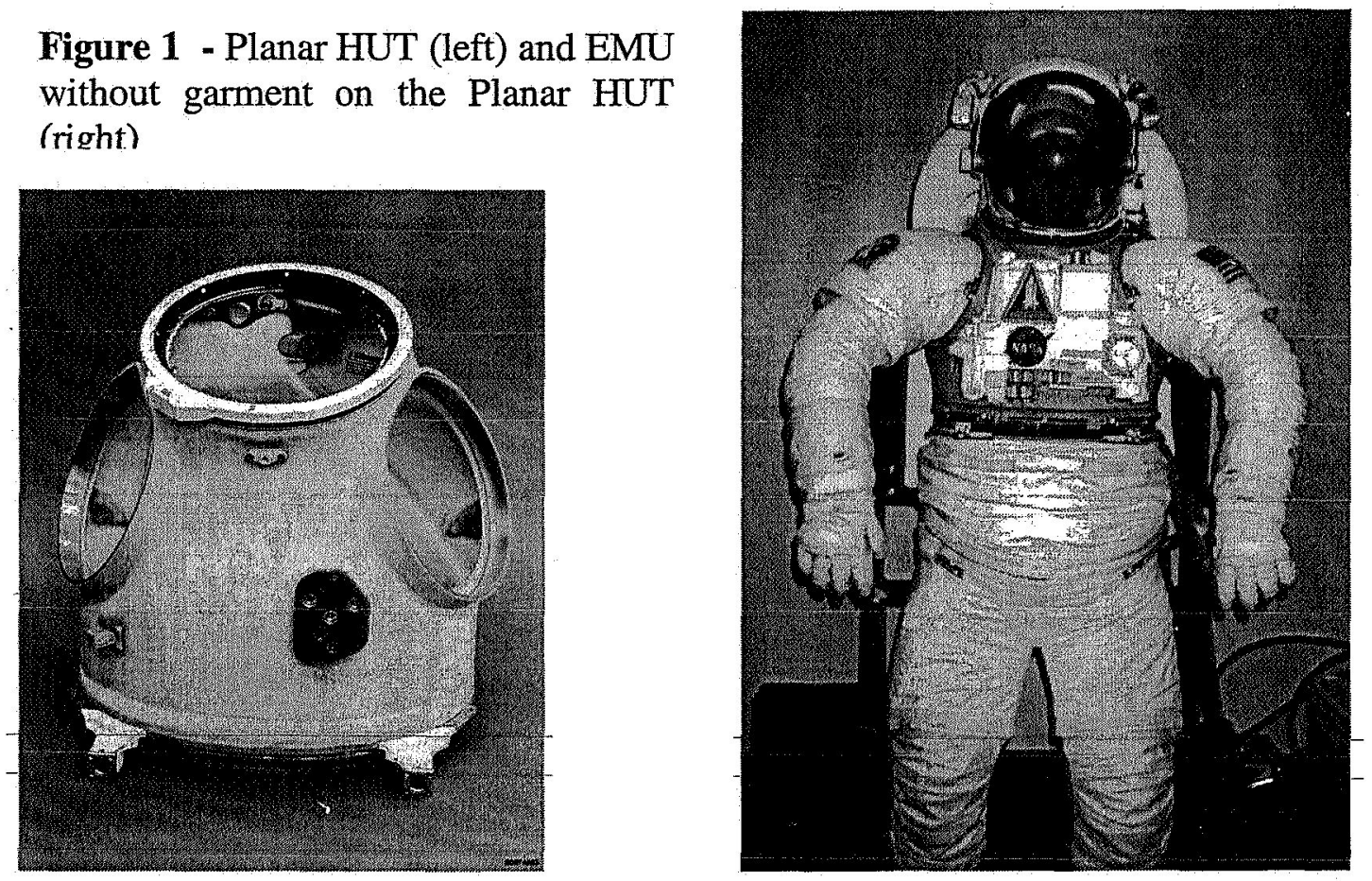

The second hardware item that received attention of the team was EVA training tools. There are a wide variety of tools used for EVA operations. As such, many training versions of these tools are utilized in the NBL for practicing EVA tasks. Many of these tools have been modified to be neutrally buoyant in the water. However, a number of have not, and it was found that several commonly used tools were among the heaviest. Many of these tools weigh between 5 and 11 pounds in the water (buoyant weight). During NBL training it is sometimes necessary for the crewmember to be holding one of these tools with arms fully extended. This activity puts the crewmember's shoulder under a high amount of stress. The duration of such an activity varied per task and the crewmember's preference.

Operations / Training: As mentioned above the NBL is utilized to:

- Evaluate an EVA candidate's skills with respect to EVA tasks and overall performance with suit mobility and translation

- Development of EVA tasks. Perform task feasibility, choreography, and fine tune timeline estimates

- Train EVA assigned crews on the mission tasks

Although a comprehensive training program is established for EVA candidates, there is a certain amount of mentor training and "on the job" training. The Crew Office had recently established an "EVA Skills" training program aimed at providing prospective EVA candidates more opportunities to learn and demonstrate generic EVA skills. The initial NBL exercises for a prospective EVA candidate were heavily task focused, meaning that there was little time set aside for suit familiarization and fundamentals of suit operation. Prior to this investigation, the first NBL experience for many candidates was considered a "weeding out" as opposed ensuring new candidates obtained optimum suit sizing, proper weigh out (achieving neutral buoyancy), familiarization with the NBL environment and other basics necessary to ensure success.

Another key operational variable is the optimum work envelope. EVA compatible hardware (i.e. worksites on the ISS, on-orbit replaceable units, tools) should be designed to allow the crewmember to work in a comfortable work envelope. Ultimately, for many missions this was not feasible because of either inadequate review of the original worksite design or changes to the worksite. There are several vital tasks that were performed on the Hubble Space Telescope and ISS that required crews to extend multiple times outside of the optimum work envelope as depicted in Figure 1. Performing such a task once or twice in orbit is not generally an issue. But repeatedly performing tasks outside of the envelope in the NBL, in a gravity environment, can be significant. The crewperson is at times fighting the torque of their own arm weight, the weight of the tool, and the torque of the suit pressure 
garment. For ISS and Hubble missions, training ratios for EVAs have ranged from 10 to 15 meaning that a crewmember may experience up to 30 runs in the NBL in an 18-month time frame. And during each run the crewmember may be repeating a questionable task multiple times. While necessary, the repetition of such physically demanding tasks during training provides an opportunity for overuse and repetitive stress injuries.

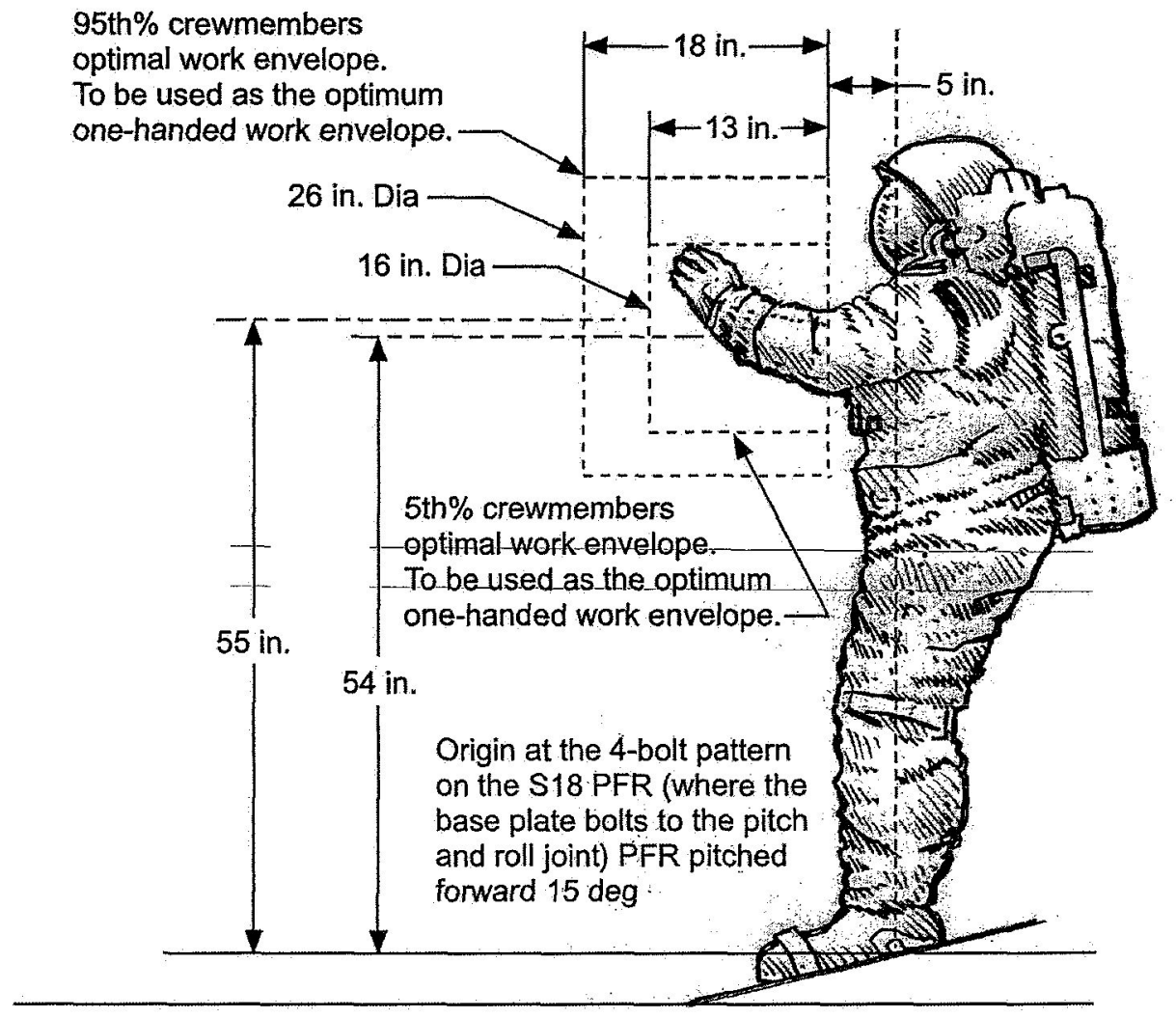

Figure 2 EMU Work Envelope (for reference only)

Finally, another important aspect in NBL training is that crews are subjected to inversion for short durations. 'Inversion' is defined as any time the body is oriented in a head down position greater than 45 degrees. Inversion for short durations can have multiple effects but the most significant with respect to this issue is the fact that the shoulders would bear the full weight of the body while inverted. Crewpersons were not provided a restraint within the suit to adequately and comfortably support the weight of the body ${ }^{\ddagger}$, so minor shoulder bruising was almost always experienced by crewmembers during NBL runs with more than a few seconds of inverted operations. The reason for inverted operation is that Space Station mock-ups, due to their size and configuration, cannot be easily rotated/maneuvered during or between training runs. In order to practice the full choreography of an EVA mission, the crewmembers must sometimes remain inverted for minutes at a time.

Table 2 summarizes only those findings that led to recommendations that are more general in nature and therefore are appropriate for a lessons learned database.

\footnotetext{
\# When the Pivoted HUT was originally designed, a shoulder harness, similar to those used on backpacks, was developed as an option to allow the crewmember to bear the weight of the EMU on his shoulders during 1-G exercises. However, it did not find favor with early EVA crewmembers and it was removed from general use in favor of location-specific padding sewn on the crewmember's cooling garment, and padding became the de facto standard for addressing pressure points and comfort issues. Subsequent EVA crewmembers were unaware that the harness existed.
} 


\section{Table 2 - Significant Findings from the Investigation}

\begin{tabular}{|c|c|}
\hline Prevalence & $\begin{array}{l}\text { - Sixty-four percent (14/22) of the surveyed EVA astronauts had experienced some degree of } \\
\text { shoulder pain that they attributed to EVA training in the EMU. } \\
\text { - The survey of EVA training-related shoulder injuries did not include all astronauts in EVA } \\
\text { training. } \\
\text { - During the interviews with EVA astronauts, it became evident that training in the EMU may } \\
\text { also result other types of injuries, including fingernail delamination, elbow pain, knee pain, } \\
\text { foot pain, and peripheral nerve compression leading to transient loss of sensation in certain } \\
\text { areas of the upper or lower extremity. }\end{array}$ \\
\hline $\begin{array}{l}\text { Prevention - } \\
\text { Supervised } \\
\text { EVA } \\
\text { Workouts }\end{array}$ & $\begin{array}{l}\text { - ISS and Space Shuttle Program (SSP) Medical Operations Requirements Documents } \\
\text { (MORDs) (SSP5260 Rev. B and SSP 13956) state that conditioning programs will be available } \\
\text { to all spaceflight crewmembers and shall be scheduled in three periods of two hours per week. } \\
\text { Crew participation was not monitored. } \\
\text { - Athletic workouts supervised by athletic trainers result in fewer training-related injuries and } \\
\text { better outcomes than unsupervised athletic training. } \\
\text { - Thirty percent }(3 / 10) \text { of the astronauts with preexisting shoulder injuries reported that they } \\
\text { were made worse by training in the NBL. }\end{array}$ \\
\hline Diagnosis and & - The NBL Physicians have begun questioning suited-crew about recent orthopedic injuries \\
\hline Treatment & $\begin{array}{l}\text { with photo-documentation of injuries as required. However they did not participate in weekly } \\
\text { "All Doc's" meetings held at the Flight medical clinic. } \\
\text { - If a crewmember does have an issue they may seek help from an ASCR representative, the } \\
\text { NBL physician or the flight surgeon. But all } 3 \text { may not know of a particular issue. } \\
\text { - The Flight Medical clinic did not have on-call orthopedic surgeon expertise available. } \\
\text { Consultant orthopedic surgeons may or may not have had familiarity with the unique aspects } \\
\text { of EVA training and mission operations }\end{array}$ \\
\hline $\begin{array}{ll}\text { Injury } & \text { \& } \\
\text { Symptom } & \\
\text { Reporting } & \end{array}$ & $\begin{array}{l}\text { - Only recently have the NBL physicians and the ASCR representatives been able to access and } \\
\text { input into the crewmember's electronic medical records. } \\
\text { - The existing system for medical surveillance monitoring of injuries did not detect the problem } \\
\text { of shoulder injuries sustained in astronaut EVA training. } \\
\text { - A protocol/forum for inter-directorate communication of medical issues associated with } \\
\text { operation of the EMU did not exist. } \\
\text { - Data collected via the observational study to identify EMU suit-related symptoms experienced } \\
\text { by all astronauts in training at the NBL has the additional benefit of providing immediate } \\
\text { insight into issues as they develop. Feedback to the FMC will enhance awareness of the } \\
\text { medical issues associated with EVA training }\end{array}$ \\
\hline $\begin{array}{l}\text { Suit Fit - } \\
\text { Measurements }\end{array}$ & $\begin{array}{l}\text { - There is no NASA-approved sizing document levied on the EMU processing contractors. The } \\
\text { most recent revision (now obsolete) does not include the Planar HUT or Space Suit Assembly } \\
\text { enhancements } \\
\text { - Differences exist in the way the EMU processing organizations take measurements. } \\
\text { Measurements are recorded in inches per procedures used by the Crew and Thermal Systems } \\
\text { Division while they are recorded in centimeters at United Space Alliance (USA)/FCE. } \\
\text { - There are numerous controlled dimensions in the design and manufacture of the HUT, but } \\
\text { none are directly relatable to crewmember measurements currently recorded. Standardized } \\
\text { landmark-based measurements may not be sufficient to size the HUT for the crewmember. }\end{array}$ \\
\hline $\begin{array}{l}\text { Suit Fit } \\
\text { Sizing }\end{array}$ & $\begin{array}{l}\text { - Current EMU sizing techniques do not account for how the human body fills individual suit } \\
\text { components. Current sizing is based on linear measurements. Instead, it should consider both } \\
\text { linear and volumetric measurements to optimize suit fit. } \\
\text { - The suit sizing algorithm, which was developed for the Pivoted HUT two decades ago, does } \\
\text { not include all relevant measurements for fitting the crew and does not reflect the sizing } \\
\text { requirements for the Planar HUT. } \\
\text { - Optimum } 1-G \text { suit fit does not necessarily correlate with optimum 0-G suit fit. In microgravity, } \\
\text { a } 5-7 \mathrm{~cm} \text { elongation of the spinal column occurs. Fluid shifts and changes in body position }\end{array}$ \\
\hline
\end{tabular}




\begin{tabular}{|c|c|}
\hline & $\begin{array}{l}\text { within the suit also occur on orbit. } \\
\text { The Planar HUT is the current flight-certified configuration of the EMU. To optimize suit fit to } \\
\text { reduce the risk of shoulder injury, some astronauts prefer the option of training in the Pivoted } \\
\text { HUT-a non-flight-certified configuration. }\end{array}$ \\
\hline $\begin{array}{l}\text { Suit Fit - } \\
\text { Padding and } \\
\text { Harnesses }\end{array}$ & $\begin{array}{l}\text { - Inexperience with the shoulder harness for the last } 14-15 \text { years during NBL training means that } \\
\text { astronauts, suit engineers, and technicians are unfamiliar with this hardware. } \\
\text { - Crewmembers are not adequately educated on different padding configurations available. They } \\
\text { have not been aware of the option to use the harness within the HUT for the last } 14-15 \text { years. } \\
\text { - A formal review of padding design was not performed with the introduction of Planar HUTs. }\end{array}$ \\
\hline Suit Design & $\begin{array}{l}\text { - The Planar HUT design uses a standardized body seal closure and scye bearing size that are } \\
\text { common to the Medium, Large, and Extra Large HUTs. The size of these components restricts } \\
\text { don/doff and mobility envelopes, and can increase the risk of shoulder injuries in some } \\
\text { crewmembers } \\
\text { - The Planar HUT shoulder design restricts the normal scapulothoracic motion of the shoulder } \\
\text { joint, resulting in rotator cuff impingement in certain arm positions. } \\
\text { - The risk of EVA training-related shoulder injury can be reduced but not eliminated by } \\
\text { redesigning the Planar HUT shoulder joint. }\end{array}$ \\
\hline \multirow[t]{3}{*}{ EVA Training } & - NBL lesson plans did not specify the limits on tasks requiring inverted body orientations, use \\
\hline & of heavy tools, or the need to perform tasks in the nominal EMU work envelope. \\
\hline & $\begin{array}{l}\text { - Lesson objectives for initial NBL runs in ASCAN EVA training were task-fucused-Providing } \\
\text { a functional fit check and assessments of an adequate weigh out were not formal initial lesson } \\
\text { objectives. } \\
\text { - Certain hardware designs and tasks force crews to overextend their arms and perform duties } \\
\text { outside of a nominal EMU work envelope. } \\
\text { - The NBL training ratio has historically exceeded } 10 \text { to } 1 \text {. The maximum number of runs } \\
\text { typically performed by any one crewmember in a week is three }\end{array}$ \\
\hline $\begin{array}{l}\text { EVA Training } \\
\text { Tools }\end{array}$ & $\begin{array}{l}\text { - Frequent use of heavy tools outside of the nominal work envelope in the NBL increase the risk } \\
\text { of shoulder injury. } \\
\text { - There are no generic requirements with respect to buoyant tool weight for use in the NBL. }\end{array}$ \\
\hline
\end{tabular}

\section{Key Recommendations and Lessons Learned}

At the beginning of this investigation it was assumed that a single discrete root cause for the prevalence of shoulder injuries would not be found. As Table 2 illustrates there is a suite of variables that together in some combination could have led to each case of shoulder injury. To address this multi-factorial problem several specific recommendations would be necessary. Over 50 recommendations were ultimately published. Of those, 27 are directly applicable towards future EVA programs or are indirectly applicable to any human space flight program. As with the findings, the recommendations highlighted in this section are provided only at a summary level. These recommendations have been categorized into three areas, those that are primarily technical in nature, those that deal with medical monitoring, and those that pertain more to the organizational and cultural aspects of the EVA organizations at JSC.

Before proceeding however the authors would like to make a few general statements on the recommendations provided by the Tiger Team. Subjectively there are a small group of these recommendations that would go a long way towards resolving this issue. These include redesign of the HUT, improving medical surveillance of the crewmembers, and improving the training and awareness of the crewmembers. A HUT redesign is problematic largely because of the fact that a redesign and fleet replacement would take several years. Over this same timeframe the bulk of the ISS assembly will be completed. This assembly will require thousands of hours in the NBL by several dozen crewmembers. In addition, a redesign of the HUT for the current configuration of the EMU was considered a large expenditure for a point solution. The authors recommended to JSC management that it would be more beneficial to spend the time and resources instead on starting efforts to ensure the next suit (planetary or low earth orbit) design would benefit from these lessons learned. The authors believe that instituting the remaining recommendations could reduce the risk of recurrence major injuries dramatically if not totally in a relatively short 
time frame. The complete list of recommendations was assigned to directorate levels of authority at JSC to ensure closure and/or future compliance.

\section{A. Technical recommendations for the Current Design}

This category of recommendations includes those that are more specific and technical in theme. These technical recommendations direct a specific corrective action but indirectly provide for lessons learned.

Develop a NASA integrated astronaut anthropometric database for sizing the EMU, the Advanced Crew Escape suit, and any other pressure or partial-pressure suit.

At JSC there is more than one contractor or government lab maintaining anthropometric data on crewmembers. Each location has a specific need and may maintain quite different measurements and algorithms used for sizing. It was determined that the best practices for measuring and maintaining up to date anthropometric data should be consolidated and maintained by the Government. This data over time can prove to be useful for other studies where statistical data on average crewmember sizes is required.

Conduct additional studies to accurately characterize spinal elongation in 0 G. Formally assess other factors affecting suit fit in micro gravity.

Integral to sizing a crewmember correctly for on-orbit operations is the adjustment made for spinal elongation experienced from prolonged exposure $0-\mathrm{G}$. It was found that spinal elongation is neither well understood nor welldocumented. Further, there is no medically accepted basis for the current values used by the several organizations responsible for sizing crewmembers for EMU other pressure suits. The Tiger Team deemed a formal study utilizing a majority of the future crewmembers assigned to flights necessary .

Assess updating the primary work envelope as defined in NSTS 07700, Vol. XIV, Appendix 7 based on latest current suit fit and population.

Update documentation (i.e. crew consensus form) to ensure evaluation of task and hardware during development NBL runs will include an assessment of the crewmember's capability to perform the task within the specified work envelope.

One of the top-level specifications at JSC for defining manned space fight requirements is NSTS 07700. This document contains an entire chapter on EVA requirements, including the definition of the EMU's primary work envelope (similar to Figure 2). The version published in NSTS 07700 fails to address the changes that occurred with the introduction of the Planar HUT. In addition, the document is used as a guideline rather than a hard requirement to which spacecraft with EVA interfaces must comply. For example, several of the ISS and Hubble "EVA compatible" worksites force the crewmembers to work outside of this envelope, in some cases for extended periods of time. Maintaining up to date specifications and conforming to those is a practice that is difficult for most programs but is key to ensuring mission success and safety.

Future NBL training tool development (all handheld tools) should require tools to be neutrally buoyant. This should be documented in the appropriate work instructions and/or general design requirements.

As described in the findings, several of the hi-fidelity NBL tools are too heavy. In some cases the desire for hifidelity training aids leads to heavier and heavier tools with little thought to long-term consequences. Although some NBL tool designs were constructed to be neutrally buoyant, there was no global requirement levied on NBL tool designers as such. Requirements governing all training tools to be used by suited crewmembers in the NBL are being changed at this time.

\section{The HUT harness and Padding shall be redesigned}

The current suit configuration does have a HUT harness and padding designed to either relieve pressure points or isolate the crewmember from the pressure points entirely (Figure 3). The adequacy of the harness and padding had 
not been formally evaluated since the original design of the Pivoted HUT. During transition to the Planar HUT and increasing frequency of training, some of this equipment began to be modified in an ad-hoc fashion. For example, thin strips of Teflon panels were added to the pads to address a "hot spot" or point load. The HUT harness (Figure 3), although available, was not used by any of the current crewmembers. Ultimately, it was determined that reexamination and reengineering of these items was necessary. It appears that this portion of the suit design was given little to no thought over the years because it was deemed a crew comfort or preference item. In fact certain items like the HUT harness had been almost erased from the corporate memory of the program
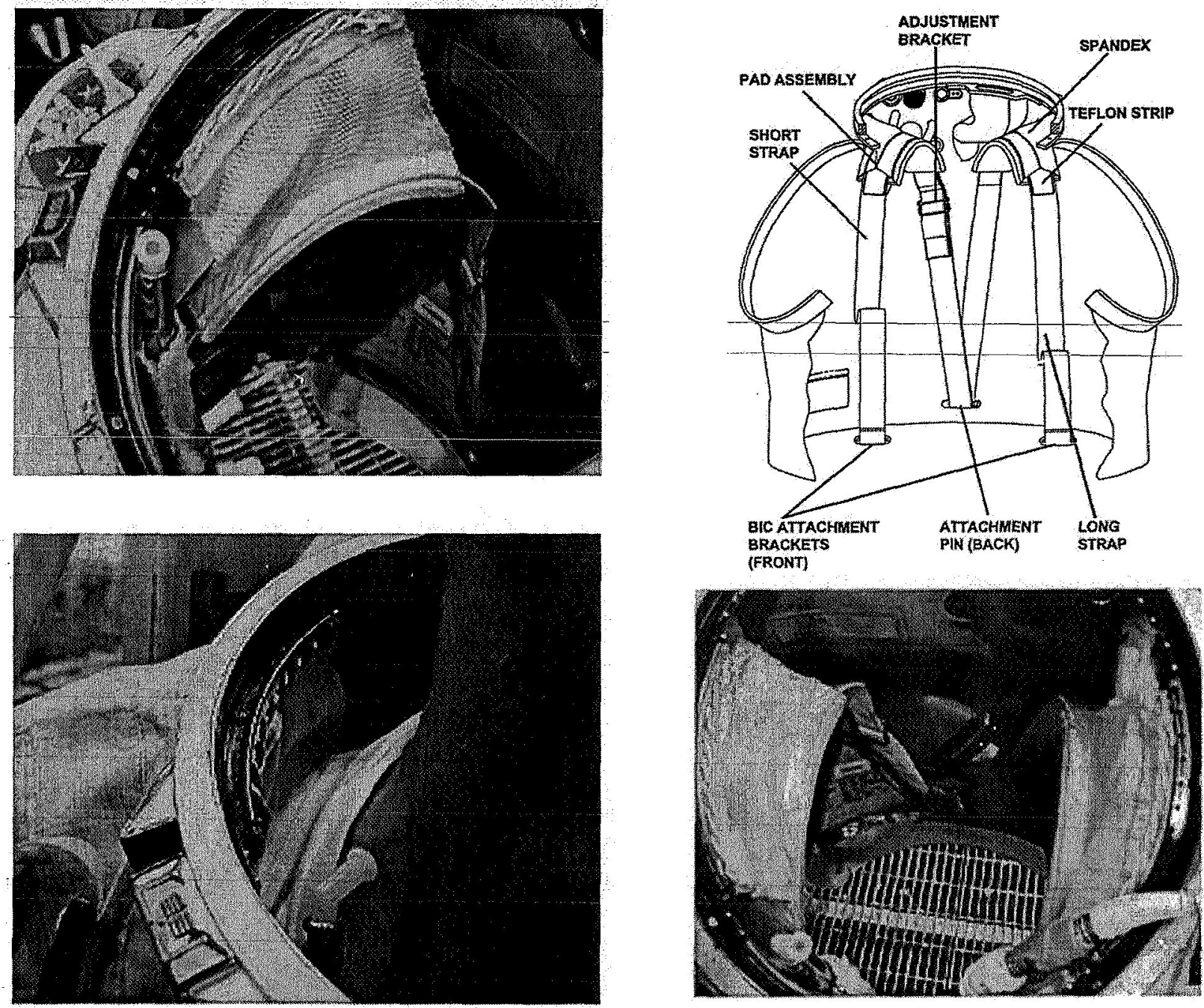

Figure 3. Original HUT Harness

Develop new suit sizing constraints, requirements, and processes. Re-institute the EMU Sizing Document (Class I controlled by NASA) and update it to reflect the latest configuration of space suit assembly hardware, suit sizing requirements and utilization of laser scanning.

The method by which suits have been sized was experimental at best in the beginning of the program. Sizing a suit had been reduced to an "art form" for some areas with this responsibility. NASA gave up control of this process several years ago and left it up to the internal processes of each area performing this task. Based on the Tiger Team evaluation it was determined that additional rigor and control was required and, as such, put forth the above recommendation.

\section{B. Health and Surveillance}


This next grouping of recommendations is related to the health and medical monitoring of the crewmembers in training. Even if the equipment and operations are designed to mitigate the potential for injury, it is the belief of the Tiger Team members that a rigorous monitoring program needs to be in place. Too many variables are outside of the control of the design and operations teams to ensure no injuries occur. Therefore, the next line of defense is to catch any potential serious injuries early.

All astronauts at each stage of EVA training must have an assessment with an ASCR to develop an individualized EVA workout. Astronauts should be referred to a designated athietic trainer or a physical therapist for a functional shoulder assessment at least four weeks prior to starting astronaut candidate (ASCAN) EVA training, EVA Skills training, and Mission-Specific EVA training.

The NBL Physician will assess each astronaut following suited runs to evaluate possible overuse syndromes, injuries, and other medical issues in astronauts. They will arrange follow-up in the Flight Medical Clinic for individuals if clinically indicated.

If clinically indicated, the NBL Physician and other physicians conducting medical evaluations of EVA crew prior to NBL training will perform a history and physical examination to detect unresolved shoulder symptoms/injuries.

The NBL Physician will assess each astronaut following suited runs to evaluate possible overuse syndromes, injuries, and other medical issues in astronauts. They will arrange follow-up in the FMC for individuals if clinically indicated.

A member of the ASCR team should perform a follow-up consultation with EVA crewmembers within 48 hours of performing an NBL run.

All of the above recommendations combine into one sentiment: constant surveillance of the health of EVA crewmembers in training is required. The Tiger Team truly believes that training in the NBL is a physically demanding task and there exists a high potential for minor injuries as a result. If left alone, some of these minor injuries could lead to serious issues. Consequently, the team recommended additional rigor and steps to improve and expand the current crewmember health surveillance program. However, the real lesson learned here is the importance of defining the responsibilities each organization has within this surveillance circle. Three separate groups (NBL Physicians, FMC doctors, and ASCRs) are independently and collectively crucial to ensuring the success of the surveillance program. It is imperative that these organizations understand where their responsibilities begin and end to most effectively undertake this collaborative effort.

\section{A retrospective study of all types of injuries associated with EVA training should be conducted}

This entire study hinged on the results from the survey. Without it many of the findings and recommendations could not be substantiated. Ultimately, it was the opinion of the Tiger Team that this type of data should always be available and periodically reviewed by the responsible parties. Without a data baseline several months were added to this investigation. New programs with intensive physical training programs need to ensure that some organization is maintaining pertinent metrics on trainee health and medical issues.

Guidelines for allowable frequency of runs any one crewmember can perform in a week (or within a meaningful time period) should be developed and documented. Criteria for allowable frequency and duration of inverted training for EVA need to be established, documented, and implemented. These criteria should include minimum rest times between inverted sessions.

EVA operations and training for the early Space Shuttle Program was much less frequent and much less challenging than that of today, which includes assembly for ISS, maintenance of Hubble and other activities. In the early days of the Shuttle Program, guidelines to protect the crew were most likely not considered necessary. With the progression to more complex programs requiring a magnitude more EVAs per year, guidelines like the two shown above are necessary. This is one of many areas where requirements creep placed more challenges on the crew, potentially pushing some to the point of serious injury. 
heavier tools (cautions, hold time constraints), need for diver assistance, cautions to be expressed to crew to limit general overexertion and for specific tasks that are historically difficult.

\section{Astronauts in the early portion of their training should be provided with lessons learned from experienced crewmembers with respect to suit fit and operations in NBL. These lessons learned should be documented (i.e. EVA Standard Operation Procedures).}

The above recommendations are in response to many of the findings that were concerned with training and risk awareness. A good defense against the recurrence of injuries is to properly train both the crew and individuals supporting the crew on the risks involved with the training. For the crew this means both physical conditioning (preventative training) and knowledge transfer. Many of the recommendations can be best summarized as raising awareness of the risks involved with training in the NBL with the current suit and tool designs. Much of the awareness training was simply passed on from one crewmember to another and from one NBL diver to another. Over time this type of knowledge transfer can become diluted and sometimes be forgotten altogether. This is why awareness training and physical training for the crew and support personnel must be documented and provided repeatedly and consistently.

\section{Conclusion - Thoughts on Future EVA Suit Development and Beyond}

A primary lesson learned towards future suit development efforts is that a suit must be designed by the right expertise with both operational and training objectives in mind. The current EMU was designed first for near earth orbit applications and only modified slightly to ensure an effective training environment. The mantra for many years has been "train what you fly in". Training in the NBL with flight-like gear has great advantages to both the crew and designers. For the crew, utilizing such a hi-fidelity trainer provides for a smooth, if not seamless, transition to actual flight experience. For the designer, utilizing flight-like hardware for NBL training provides an opportunity to acquire thousands of hours of operation in one year on a design that may only see a one to two hundred hours in space over several years. This opportunity provides a "wringing out" for the hardware and, in the case of the EMU, the NBL experience has been invaluable in early identification of design shortcomings. But these advantages need to be weighed against disadvantages of operating in a sub-optimal 1-g design. A crewmember will spend an order of magnitude more time in a space suit on the ground than he/she will on-orbit. This amount of exposure to a particular design (and its 1-g shortcomings) means future EMU and EVA tool engineers must design for training as well.

Another key lesson is that biomechanical engineers, kinesiologists, and orthopedic specialists should participate during the preliminary design phase of future suit efforts to best optimize the suit fit from an ergonomic perspective. Additionally, laser anthropometric studies of male and female astronauts, biomechanical analysis of shoulder joint motion in both genders, use of CAD models of shoulder joint motion and EMU shoulder joint design should all be incorporated into the development of the next-generation space suit.

As was witnessed by our experience with the EMU, once hardware as critical and complicated as a space suit has been designed, certified and fabricated it becomes increasingly difficult - if not impossible - to make adjustments to improve the human to machine interface. This is especially true in the case of a space suit where the interfaces are fine-tuned to balance the needs of several competing requirements. Because of the high expense of the design, certification and implementation process for new hardware, NASA plans to continue to fly the existing EMU design to support Shuttle and ISS assembly operations for the next 8 years. Future suit designs must plan and allocate enough resources in the design phase to ensure a more "biomechanically friendly" suit, which allows for mobility within a nominal work envelope that will not obstruct normal human motion and not require increased strength and endurance to operate. Future suit designers must also attempt to broaden on the anthropometric range of the current suit. Finally, future programs must ensure that adequate requirements and processes are in place to address many of the other recommendations suggested in this paper in the areas of training, awareness, communication, crewmember conditioning, and medical surveillance.

Finally many of the adverse findings in this study are the direct result of requirements creep and a slow disintegration of corporate memory. Leaders of future space flight programs with design lifetimes of 10 years or 
more must strive to put in place controls for all critical processes and develop knowledge capture systems to ensure the rationale behind many of the early program decisions is maintained throughout the program history. Programs that put hardware in intimate contact with humans such as a space suit must have a broader definition of critical process or requirements because any slight change or variance can directly affect the crewmember's long term health and safety. 


\section{Acknowledgments}

The authors would like to acknowledge the extremely helpful efforts of several individuals who supported the original Tiger Team and provided their expertise towards a rather difficult issue.

\begin{tabular}{|c|c|}
\hline Mission Operations & DX/Mary Fitts, Zane Ney \\
\hline Crew Systems & EC/Joe Kosmo \\
\hline Space Suit Assembly Subsystem & EC5/Scott Cupples \\
\hline Astronaut Office & $\mathrm{CB} /$ Rex Walheim \\
\hline Human Factors (ABF) & SF3/Sudhakar Rajulu and Luis Gonzalez \\
\hline Flight Crew Engineering & $\begin{array}{l}\text { USA/FCE/Don Campbell, Lou Carfagno and Chris } \\
\text { Trevino }\end{array}$ \\
\hline Flight Medicine & SD2/Joe Dervay, Rick McCluskey \\
\hline Occupational Health / NBLPhysician & SD/Chuck Ross and Sam Strauss \\
\hline $\begin{array}{l}\text { Astronaut-Strengthening,-Conditioning, } \\
\text { and Rehabilitation Team }\end{array}$ & SD/Jamie-Chauvin, Beth-Shepherd, and-Gorey-Twine \\
\hline
\end{tabular}

\section{References}

${ }^{1}$ Williams, D. R., and Johnson, B.J., "EMU Shoulder Injury Tiger Team Report," NASA/TM-2003-212058, 2003. 


\section{Organizational / Cultural}

This final category of recommendations includes those that are more programmatic in nature. Core to many issues is a breakdown of communications at some level. Another key area in every program is the identification and community awareness of risks. It is crucial that all individuals working with potentially risky hardware have comprehensive training and awareness of those risks.

The NBL Physician should participate in the weekly All Docs FMC Meetings to update the FMC physicians on EMU-related medical events at the NBL.

Incorporate ASCR Assessments and Interventions into the Flight Medical Clinic Electronic Medical Record and provide updates to the Flight Surgeon weekly. Provide the NBL Physician with access to the EMR and ensure that medical assessments of astronauts at the NBL are incorporated into the EMR.

Immediately implement an integrated system of recording EVA training-related symptoms and injuries. Track this information epidemiologically and report it monthly to the FMC.

Identify representatives from Space and Life Sciences, EVA Office, Crew Office, and Safety \& Mission Assurance to establish a team to review medical issues associated with the use of EMU hardware. This group shall establish a mechanism for disseminating information, assessing risks, and achieving consensus on medical issues associated with operation of the EMU and EMU-related hardware.

Many of the findings suggested that overall communication of crew health issues needed improvement. The above recommendations and others in the full report suggest how the current organizations can start communicate more effectively when it concerns the health of crewmembers. There are obvious issues with sharing medical data pertinent to individuals; however there must be avenues established for doctors, trainers, and hardware designers to discuss medical issues.

Document EVA-specific exercises (aerobic, strength, flexibility, rotator cuff) and make them available on line.

Provide a presentation to the Astronaut Office on the benefits of ASCR-supervised athletic training and rehabilitation, with particular emphasis on conditioning strategies to reduce the risk of injury during EVA training.

Astronauts in any phase of EVA training are expected to attend scheduled fitness assessments and physical training sessions. CB management should reassess the policy regarding astronaut compliance with scheduled training.

The NBL Physician, Flight Surgeons, and ASCRs should receive additional training in the prevention, early diagnosis, and treatment of rotator cuff injuries.

Update the crew options document and processes to ensure consistent identification and training for crew options.

Conduct awareness training of the shoulder harness and initiate updates to the design to improve fit and load alleviation capability.

The EVA Branch in association with the suit engineers should develop a standardized suit fit briefing reflecting the lessons learned by experienced EVA astronauts and suit engineers. This brief should be incorporated into the EVA training flow.

NBL lesson plans need to be updated to address the following: NBL/Suit mobility familiarization (first NBL run) including a functional fit check, constraints and information related to inverted operations, use of 